\title{
Incorporación de estructuras cooperativas a un proyecto de trabajo internivel: reflexiones para la mejora educativa
}

Recibido: 15 de septiembre de 2018 / Revisado: 15 de octubre de 2018

Aceptado: 27 de noviembre de 2018 / Publicado: 19 de diciembre de 2018

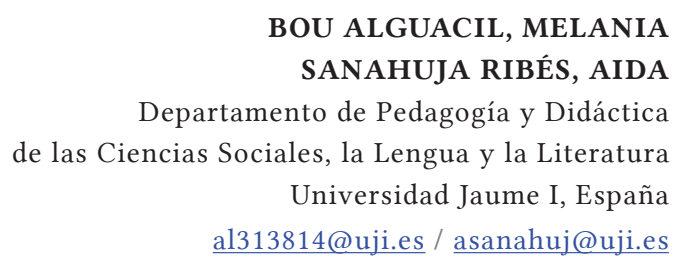

DOI: $10.24310 /$ IJNE1.1.2018.4954 FALTA

\section{RESUMEN}

El siguiente trabajo se basa en una propuesta de investigación-acción en la que se tiene por objeto principal incorporar estructuras cooperativas en un aula de infantil (3 años), mediante un proyecto de trabajo internivel desarrollado junto al alumnado de segundo de primaria. La acción se llevó a cabo en un aula de un centro público de Castellón de la Plana (España). En primer lugar se recogió información mediante un anecdotario, se formuló una entrevista a la maestra del aula y se preparó una tabla de observación con indicadores extraídos de la Guía CEIN para la Construcción de la Escuela Intercultural e Inclusiva, para comprobar el grado de cooperación. Con ello, se detectaron las necesidades y el problema de estudio. En segundo lugar, se puso en práctica el proyecto "La vuelta al mundo en 80 días", en este trabajo se presta especial atención a las técnicas de: la tutorización, el folio giratorio, una adaptación de

\section{ABSTRACT}

Incorporation of Cooperative Structures in an Interlevel Project: Reflections on Educational Improvement

The following work is based on a research-action proposal whose main aim is to incorporate cooperative structures within a preschool classroom (3 years old), through an inter-level project developed together with the primary school second grade students. The action was conducted in a public school classroom in Castellón de la Plana (Spain). Firstly, information was gathered by means of a collection of anecdotes, some interview questions were formulated for the classroom teacher, and an observation checklist was created with indicators from the CEIN Guide for the Inclusive Intercultural School Construction in order to observe the degree of cooperation. Thus, the needs and the problem of study were detected. Secondly, the Project "Around the World 
la técnica 1-2-4 y el "Juego-Concurso de De Vries". Para realizar el seguimiento de la acción y evaluar el proceso, se utilizó la observación participante, una segunda entrevista a la maestra y un análisis documental. Además, se llevó a cabo la técnica 'Felicito, critico, propongo' con el alumnado. Por último, a partir de los resultados obtenidos se extraen algunos factores y elementos que no han funcionado en la práctica abordada.

Palabras Clave: Escuela inclusiva, Practica educativa, Educación primaria, Educación infantil. in 80 Days" was implemented. In this work, special attention is given to the techniques of: tutoring, rotating sheet, an adaptation of the technique 1-2-4, and the "De Vries' Game-Contest". For the monitoring of the action and the evaluation of the process, we used the participant observation method, a second interview for the teacher, and a documentary analysis. In addition, we used the technique "I congratulate, I criticise, I propose" with the students. Finally, from the results obtained, we identified some factors and elements that have not functioned in the practise performed.

Keywords: Inclusive Schools, Educational Practices, Primary Education, Preschool Education.

\section{Introducción}

Pese a que, hoy en día, es muy común en el ámbito educativo oír hablar de educación inclusiva, a menudo, parece que dicho constructo no tiene para todos el mismo significado o las mismas connotaciones. Según indican Echeita y Ainscow (2011), nos encontramos ante un término confuso y difuso. Puesto que, según estos autores, algunas personas asocian el concepto al hecho de que el alumnado con discapacidad o diversidad funcional esté presente en un centro ordinario, mientras que otros lo entendemos en un sentido más amplio, esto es, partiendo de una lógica de heterogeneidad (reconocimiento y valoración positiva de la diversidad en el aula). Siguiendo con la misma idea Echeita (2013, p. 105) asocia la educación inclusiva a un poliedro con varias caras:

Adentrándonos en su significado lo primero que ha de señalarse es que hablar de inclusión educativa (como de su antónimo exclusión educativa), hace referencia a un concepto y a una práctica poliédrica, esto es, con muchas facetas o planos, cada uno de los cuales tiene algo de la esencia de su significado, pero que no lo agota en su totalidad.

Para ahondar brevemente en el concepto de escuela o educación inclusiva se presentan en la Tabla 1, algunas definiciones del vocablo (Barrio, 2008; Pujolàs, 2008; Ministerio de Educación, Cultura y Deporte, 2011; Cerdá e Iyanga, 2013; Muntaner, 2013). 
Tabla 1. Algunas definiciones sobre educación inclusiva.

\begin{tabular}{|c|c|}
\hline AUTOR & DEFINICIÓN \\
\hline $\begin{array}{c}\text { Barrio } \\
(2008, p .16)\end{array}$ & $\begin{array}{l}\text { "El término de escuela inclusiva hace referencia a la idea de que la escuela es para } \\
\text { todos, la educación es para todos, con independencia de las características y diferen- } \\
\text { cias de cada uno, sean éstas por razón de cultura, raza, religión, lengua, capacidad, } \\
\text { etc. Nos encontramos entonces ante una educación y una escuela de la diversidad, } \\
\text { apreciándose ésta como un deseo de que nadie sea excluido". }\end{array}$ \\
\hline $\begin{array}{c}\text { Pujolàs } \\
(2008, \text { p.37) }\end{array}$ & $\begin{array}{l}\text { "La educación inclusiva debe entenderse como un reto, no como un problema irreso- } \\
\text { luble: es normal que los alumnos sean diferentes; por lo tanto, más que preocuparnos } \\
\text { por cómo podemos conseguir grupos homogéneos (para reducir o "anular" las dife- } \\
\text { rencias), es mucho más lógico y coherente preocuparnos por cómo podemos gestionar } \\
\text { la heterogeneidad, cómo podemos enseñarles juntos a pesar de sus diferencias". }\end{array}$ \\
\hline $\begin{array}{l}\text { Ministerio de Edu- } \\
\text { cación, Cultura y } \\
\text { Deporte (2011) }\end{array}$ & $\begin{array}{l}\text { "La educación inclusiva tiene como propósito prestar una atención educativa que fa- } \\
\text { vorezca el máximo desarrollo posible de todo el alumnado y la cohesión de todos los } \\
\text { miembros de la comunidad. } \\
\text { La comunidad educativa está integrada por todas las personas relacionadas con el cen- } \\
\text { tro: alumnos, profesores, familias, otros profesionales que trabajan en el centro, ad- } \\
\text { ministración educativa, administración local, instituciones y organizaciones sociales. } \\
\text { Todos los componentes de la comunidad educativa colaboran para ofrecer una edu- } \\
\text { cación de calidad y garantizar la igualdad de oportunidades a todo el alumnado para } \\
\text { participar en un proceso de aprendizaje permanente". }\end{array}$ \\
\hline $\begin{array}{c}\text { Cerdá e Iyanga } \\
(2013, \text { p.161) }\end{array}$ & $\begin{array}{l}\text { "Todos vivimos en la misma sociedad y la escuela los prepara personal, social y pro- } \\
\text { fesionalmente para ella, por esto, no hay razón de separarlos; ya que cuando sean } \\
\text { adultos tendrán que estar juntos, compartiendo aficiones, trabajo y relaciones". }\end{array}$ \\
\hline $\begin{array}{c}\text { Muntaner } \\
(2013, \text { p.36) }\end{array}$ & $\begin{array}{l}\text { "Modelo que nos permite el planteamiento de una educación de calidad para todos, } \\
\text { basada en la equidad y la participación, con el objetivo último de evitar la exclusión y } \\
\text { la discriminación de los estudiantes por cualquier causa, situación o motivo". }\end{array}$ \\
\hline
\end{tabular}

Notemos como para Barrio (2008) y Pujolàs (2008) la educación o la escuela inclusiva es sinónimo de la escuela de la diversidad. Dichos estudiosos recogen de forma explícita en su definición el valor de la diversidad o de las diferencias existentes entre los individuos, convirtiéndose en un reto o desafío para el docente, quien debe ser capaz de gestionar de manera adecuada la heterogeneidad presente en su aula (Pujolàs, 2008), con el propósito de evitar la segregación o exclusión del alumnado (Cerdá e Iyanga, 2013; Muntaner, 2013). Conviene subrayar que la educación inclusiva compete a todos los miembros de la comunidad (Ministerio de Educación, Cultura y Deporte, 2011). 
Partiendo de esa idea de diversidad o diferencia emerge con fuerza el concepto de diferenciación pedagógica desde una perspectiva inclusiva, el cual tiene una larga tradición de estudio en el contexto anglófono (Kline, 2015; Gregory y Chapman, 2013; Bender, 2012; Campbell, 2008; Levy, 2008; Subban, 2006; Tomlinson, 2005; Heacox, 2002; Forsten, Grant y Hollas, 2002) y francófono (Prud'Homme et al, 2016; Saint-Laurent, 2007; Guay, Legault y Germain, 2006; Legendre, 2005; Caron, 2003; Perrenoud, 1996; Meirieu, 1996; Legrand, 1995; Aylwin, 1992). Dicho concepto no ha proliferado en el contexto hispánico en el sentido y las connotaciones que lo ha hecho en ambos contextos mencionados. Una de sus causas puede deberse a la asociación de educación diferenciada por sexos (single-sex). La diferenciación pedagógica desde una perspectiva inclusiva vincula y entremezcla los principios imperantes entre la educación inclusiva y la educación democrática (Sanahuja, 2017). Así mismo, un estudio de Fillion, Bergeron, Prud'homme y Traver (2016) traza la convergencia y la interdependencia entre la inclusión escolar, la educación ciudadana y la diferenciación pedagógica.

Pero, llegados a este punto nos podemos preguntar: ¿cómo podemos materializar, en nuestras aulas, los principios y valores imperantes en la educación inclusiva? En palabras de Lira (2010), las metodologías más activas, en la que el alumnado adopta un rol protagonista, se convierten en estrategias promotoras y facilitadoras del pensamiento crítico, en tanto y cuanto favorecen la comunicación de los alumnos mediante la utilización de mecanismos participativos. Se conocen varios enfoques metodológicos que favorecen y promueven tanto la gestión pedagógica de la diversidad (Bergeron, 2015; Pujolàs, 2008; Tomlinson, 2005; Sanahuja, 2017) como la gestión democrática del aula (Apple y Beane, 1999; Feito, 2010; Feito y López, 2008; Sanahuja, 2017). Actualmente, se cuenta con diferentes prácticas de aula ilustradas que permiten avanzar hacia dichos presupuestos teóricos, por ejemplo: el aprendizaje dialógico con los grupos interactivos (Elboj y Niemelä, 2010; Sanahuja, 2014) o las tertulias literarias dialógicas (Sanahuja, Benet y Traver, 2017), el enfoque propuesto desde las inteligencias múltiples (Gardner, 1983), el Aprendizaje Basado en Problemas (Araujo y Sastre, 2008), el Aprendizaje-Servicio (Batlle, 2013; Sanahuja y Moliner, 2018), los rincones de trabajo (Benet, Sanahuja y Moliner, 2016; Jiménez y Vila, 1999), los proyectos de trabajo (Moliner y Sanahuja, 2015; Sanahuja, Peiro y Piquer, 2015; López y Lacueva, 2007), el aprendizaje cooperativo (Mayordomo y Onrubia 2015; Traver, 2000; Pujolàs, 2008; Moliner, Moliner, Sanahuja y Sanmateo, 2015; Rué, 1998). En este trabajo centramos la atención en el aprendizaje cooperativo y los proyectos de trabajo puesto que en dichas metodologías se apoyan los datos empíricos que se presenta.

Así pues, podemos decir que para llevar a cabo una educación que sea inclusiva, es importante que se desarrolle un aprendizaje basado en la cooperatividad, de esta manera todo el alumnado saldrá beneficiado.

Kyndt et al. (2014) [citado en Mayordomo y Onrubia, 2015], tras los resultados de varios de sus estudios realizados, confirman que: "los alumnos realmente aprenden más cuando trabajan juntos que cuando trabajan solos". 
En la misma línea Pujolàs (2008, p.24) afirma que:

"La única manera de atender juntos en una misma aula a alumnos diferentes — tal como exige la opción por una escuela inclusiva - es introducir en ella una estructura de aprendizaje cooperativa. Así pues, sólo pueden aprender juntos alumnos diferentes (en capacidad, interés, motivación, cultura, lengua, origen social...) en una clase organizada cooperativamente, donde todos colaboran, cooperan se ayudan para alcanzar el objetivo común de progresar en el aprendizaje, cada uno hasta el máximo de sus posibilidades. Por tanto, la mayoría de personas aprenden más y mejor cuando participan en actividades con otras personas, gracias al estímulo intelectual que esto supone y la confianza que les da la ayuda que los demás pueden dispensarles si aprenden juntos”.

A pesar de las numerosas técnicas cooperativas existentes (véase Pujolàs y Lago, 2002), en la Tabla 2 , únicamente se presentan algunas de las que se abordan posteriormente en la propuesta de acción.

Una de las maneras óptimas de abordar un aprendizaje cooperativo en las aulas es mediante el trabajo por proyectos. Partimos de que la metodología de Proyectos de Trabajo, según indican Balongo y Mérida (2016), se basa en el respeto de los intereses del alumnado y se caracteriza por estar centrada en la exploración e indagación del entorno. Los Proyectos de Trabajo se apoyan en procesos de trabajo cooperativo y propician ambientes globales y flexibles de aprendizaje. Por su parte, Ruiz y Mérida (2014) indican que los Proyectos de Trabajo no solo favorecen la participación del alumnado y del docente, sino que consideran de vital importancia la implicación de todos los miembros de la comunidad educativa, especialmente la colaboración de las familias.

El principal propósito de este trabajo se basa en presentar una práctica educativa realizada conjuntamente entre la clase de infantil 3 años con la clase de $2^{\circ}$ de educación primaria. Con el proyecto realizado se ha pretendido fomentar la cooperación entre el alumnado, encaminándonos así, hacia una educación más inclusiva. Por ello, mediante este proyecto se quiere que el alumnado de 3 años se inicie en el trabajo cooperativo, para así desarrollar ciertas habilidades y detectar los beneficios que se obtienen trabajando de esta manera.

Tabla 2. Breve definición de algunas técnicas cooperativas.

\begin{tabular}{|c|l|}
\hline TÉCNICA & \multicolumn{1}{c|}{ BREVE DEFINICIÓN } \\
\hline \multirow{3}{*}{ Tutorización } & $\begin{array}{l}\text { Esta técnica consiste en tutorizar, formando parejas de alumnos, con relaciones asimétricas } \\
\text { (uno es tutor y el otro tutorado). Ambos tienen un objetivo común, conocido y compartido (la } \\
\text { enseñanza y el aprendizaje de contenidos a partir de una interacción estructurada), y comporta } \\
\text { ventajas tanto para el tutor, como para el tutorado. Por un lado, el tutor adquiere un dominio } \\
\text { profundo de los contenidos, ya que los tiene que enseñar al tutorado, y mejora en autoestima } \\
\text { y responsabilidad. Y, por otro lado, el tutorado se beneficia de un aprendizaje individualizado, } \\
\text { con ayuda ajustada a sus necesidades (Durán, 2004). }\end{array}$ \\
\hline
\end{tabular}




\begin{tabular}{|c|c|}
\hline Folio giratorio & $\begin{array}{l}\text { El educador o la educadora asigna una tarea a los equipos de base (una lista de palabras, la } \\
\text { redacción de un cuento, las cosas que saben de un determinado tema para conocer sus ideas } \\
\text { previas, una frase que resuma una idea fundamental del texto que han leído o del tema que } \\
\text { han estado estudiando, etc.) y un miembro del equipo empieza a escribir su parte o su apor- } \\
\text { tación en un folio «giratorio». A continuación, lo pasa al compañero de al lado siguiendo la } \\
\text { dirección de las agujas del reloj para que escriba su parte de la tarea en el folio, y así suce- } \\
\text { sivamente hasta que todos los miembros del equipo han participado en la resolución de la } \\
\text { tarea. (Pujolàs y Lago, 2002, p.88) }\end{array}$ \\
\hline $1-2-4$ & $\begin{array}{l}\text { El maestro o la maestra plantea una pregunta o una cuestión a todo el grupo, por ejemplo, } \\
\text { para comprobar hasta qué punto han entendido la explicación que acaba de hacerles, o bien } \\
\text { para practicar algo que les acaba de explicar. El maestro o la maestra facilita a cada partici- } \\
\text { pante una plantilla, con tres recuadros (uno para la "situación 1"; otro para la "situación 2"; } \\
\text { y otro para la "situación 4"), para que anoten en ella las sucesivas respuestas. } \\
\text { Dentro de un equipo de base, primero cada uno (“situación 1") piensa cuál es la respuesta } \\
\text { correcta a la pregunta que ha planteado el maestro o la maestra, y la anota en el primer recua- } \\
\text { dro. En segundo lugar, se ponen de dos en dos ("situación 2"), intercambian sus respuestas } \\
\text { y las comentan, y de dos hacen una, y la anotan, cada uno, en el segundo recuadro. En tercer } \\
\text { lugar, todo el equipo (“situación 4"), después de haberse enseñado las respuestas dadas por } \\
\text { las dos «parejas» del equipo, han de componer entre todos la respuesta más adecuada a la } \\
\text { pregunta que se les ha planteado. (Pujolàs y Lago, 2002, p.88) }\end{array}$ \\
\hline $\begin{array}{c}\text { Juego- } \\
\text { Concurso } \\
\text { de De Vries }\end{array}$ & $\begin{array}{l}\text { Basándose prioritariamente en la cooperación, esta técnica también combina elementos com- } \\
\text { petitivos, ya que en un momento de su desarrollo, los grupos heterogéneos competirán entre } \\
\text { ellos. Se trata de que los estudiantes aprendan a competir entre ellos de una forma "sana", } \\
\text { a través del trabajo cooperativo. Ofrece la ventaja de poder trabajar contenidos de materias } \\
\text { de una forma divertida a la vez que aprenden a mejorar sus relaciones interpersonales, a } \\
\text { integrarse mejor en el grupo, a reconocer y valorar los esfuerzos realizados por cada uno de } \\
\text { los miembros de los equipos, etc. Los estudiantes son asignados a grupos de } 4 \text { a } 6 \text { miembros, } \\
\text { heterogéneos (Santos, s.f.,p.27). }\end{array}$ \\
\hline
\end{tabular}

\section{Diseño y metodología}

\subsection{Método}

Centrándose en los parámetros de la investigación cualitativa (Sandín, 2003), se presenta un estudio de caso (Stake, 1998) que trata de describir un proceso de investigación-acción (Moliner, Sanahuja y Benet, 2017; Latorre, 2003) llevado a cabo con el propósito de introducir estructuras de aprendizaje cooperativo en un aula de infantil 3 años a través de un proyecto de trabajo articulado junto a una clase de $2^{\circ}$ de educación primaria. 


\subsection{Contexto y participantes}

El centro en el que se ha llevado a cabo la experiencia educativa que aquí se presenta, es de carácter público y abarca infantil y primaria. En el curso académico 2017/18 se ha establecido por primera vez el horario de jornada continua (de 9 h a 14 h). Está formado por tres aulas por curso, habiendo así, 9 aulas en infantil y 18 en primaria, más un aula de Comunicación y Lenguaje $(\mathrm{CyL})$. Se encuentra ubicado en una zona con buena comunicación y fácil acceso, y muy poblada. El tipo de familias del alumnado que acude al centro está constituidas por modelos nucleares, y la mayoría de estas tienen trabajo y estudios. La lengua vehicular del centro es el valenciano, aunque la mayoría de alumnado es castellanohablante. Además, se ha implantado el Programa de Educación Plurilingüe de la Consejería de Educación (Comunidad Valenciana). La metodología imperante en el centro se caracteriza por ser activa y participativa, con un enfoque globalizador, buscando constantemente la motivación, la atención a la diversidad y la evaluación de todos los aspectos del proceso educativo. También, se fomenta la solidaridad, el respeto y el compañerismo, busca la igualdad de oportunidades y promueve actividades relacionadas con los temas: paz, justicia, diferencias personales, educación no sexista, etc.

Los participantes en este estudio han sido 23 alumnos (14 niños y 9 niñas) de un centro perteneciente a la localidad de Castellón de la Plana, de la clase de infantil 3 años. Y, además, participaron también 22 alumnos ( 9 niños y 13 niñas) de $2^{\circ}$ de primaria. Cabe señalar que al tratarse de un estudio realizado en un aula real, no se revelará ningún dato que pueda identificar a sus participantes ${ }^{1}$.

En cuanto a la clase de infantil, decir que a la mayoría de los alumnos les cuesta mantener la atención y estar concentrados, es una clase muy activa, que necesita mucho movimiento. Por lo que refiere al alumnado de primaria, es una clase muy participativa y trabajadora, aunque en algunas ocasiones les cuesta seguir el ritmo de trabajo. En el aula de primaria emplean técnicas cooperativas (como el folio giratorio o grupos de investigación), para la realización de algunas actividades, y están dispuestos en grupos de 3-4 en los cuales cada alumno tiene un rol asignado (portavoz, moderador, secretario y/o encargado).

\section{Proceso de Investigación-Acción}

\subsection{Detección de necesidades (diagnóstico situacional)}

Al comienzo del proceso de investigación-acción se utilizaron una serie de instrumentos y técnicas, con el propósito de realizar un diagnóstico situacional. A continuación, se explican de manera breve la entrevista inicial con la docente, el anecdotario o notas de campo y la observación científica.

1 En cumplimiento del Reglamento 2016/679 del Parlamento Europeo y del Consejo de 27 de abril de 2016, referente a la protección de las personas físicas en cuanto al tratamiento de datos personales y a la libre circulación de estos datos, y de la Ley Orgánica 15/1999 de Protección de Datos de Carácter Personal. 
La entrevista es una técnica característica de la investigación cualitativa que permite recabar información a través de la formulación de diferentes cuestiones a los agentes implicados en una determinada realidad educativa (Ruiz Olabuénaga, 2012). Concretamente, se efectuó una entrevista semiestructurada, la cual según Báez (2009) parte de la formulación de algunos contenidos preestablecidos, pero deja abierta la posibilidad de comentar otros aspectos relevantes que van surgiendo a lo largo de la entrevista. Así pues, para llevar a cabo la detección de necesidades, se realizó una entrevista inicial a la maestra del aula en la que se efectuó la propuesta. En dicha entrevista, se preguntó por los diferentes agrupamientos utilizados, la metodología imperante en la clase, la gestión del aula y el modo de realizar el proceso de evaluación.

Además, la entrevista se completó con un anecdotario o notas de campo y una tabla de observación con diferentes ítems. Los ítems fueron adaptados de la Guía CEIN para la Construcción de la Escuela Intercultural e Inclusiva (Sales, Moliner y Traver, 2010). Conviene recordar que la observación permite contemplar detalladamente el fenómeno que se quiere estudiar seleccionando los datos o sucesos relevantes para su posterior análisis (Vázquez, 2004).

Con los datos recabados en la fase de la detección de necesidades se pudo comprobar que en el aula de infantil 3 años el alumnado iba trabajando a través de diferentes modalidades de agrupamientos (asamblea, equipos, parejas, rincones, gran grupo, etc.), pero no se utilizaba ninguna técnica cooperativa en el aula. Con el propósito de seguir avanzando e introducir el aprendizaje cooperativo en el aula, se optó por plantear el uso de algunas técnicas cooperativas. Por este motivo, se incorporaron varias estructuras cooperativas al proyecto internivel que se iba a realizar junto al alumnado del aula de $2^{\circ}$ de educación primaria.

\subsection{Propuesta de actuación}

\subsubsection{Planteamiento de objetivos}

El principal objetivo de este estudio es analizar la incorporación de estructuras cooperativas a un proyecto de trabajo internivel desarrollado con alumnado de infantil y primaria.

Dicho objetivo general se concreta en los siguientes objetivos específicos:

1. Iniciar al alumnado de infantil en el uso de técnicas cooperativas.

2. Aumentar la interacción entre el alumnado de diferentes aulas del centro.

3. Que el alumnado reconozca y valore positivamente la diversidad de sus compañeros/as.

\subsubsection{Desarrollo de las sesiones}

Por lo que respecta a la propuesta de acción, el planteamiento elaborado se desprende de la detección de las necesidades realizada, ya que se pudo observar que en el aula trabajaban a partir de diferentes mo- 
dalidades de agrupamientos y se quería incorporar algunas técnicas de aprendizaje con el fin de enriquecer y ampliar las habilidades cooperativas y las interacciones entre el alumnado. Por lo que, finalmente, se introdujeron algunas técnicas propias del aprendizaje cooperativo mediante el trabajo por proyectos.

El proyecto internivel se llevó a cabo durante el segundo y el tercer trimestre del curso académico 2017/2018. Constó de cuatro horas semanales dentro del horario del aula de infantil, aprovechando la sesión del miércoles o la del viernes (según disponibilidad horaria del grupo de primaria), generalmente para hacer las actividades conjuntas entre las dos aulas mencionadas, juntándose así, mínimo una sesión a la semana. Por tanto, había algunas sesiones del proyecto que se trabajaba solamente con el alumnado de infantil y otras sesiones (mínimo una hora a la semana) se trabajaba conjuntamente en las dos aulas mencionadas.

El eje conductor del proyecto fue "La vuelta al mundo en 80 días", siguiendo el recorrido de la historia del libro: La vuelta al mundo en 80 días de Jules Verne (Ed: Bromera. Versión: Jesús Cortés).

Conviene apuntar que por cuestiones de espacio no se explicarán todas las sesiones que conforman el proyecto mencionado, sino que atendiendo al objetivo planteado en este estudio solamente se presentarán las sesiones en las que se han introducido diferentes técnicas propias del aprendizaje cooperativo. Seguidamente, en la Tabla 3, se presenta, de manera resumida, una descripción de dichas sesiones.

Tabla 3. Descripción de las diferentes técnicas implementadas

\section{Tutorización ${ }^{2}$}

Para hacer esta actividad, el alumnado de la clase de infantil 3 años se juntó con el alumnado del aula de primaria para escribir una nota en la que se comunicaba que a la semana siguiente estudiarían "Italia", dirigida a las familias, pidiendo el material que se necesitaba para realizar una actividad en los próximos días. El alumnado de infantil únicamente, debido a que se están iniciando en procesos de escritura, debió de intentar escribir dos palabras (Italia dos veces) con ayuda de los mayores, y su nombre. La nota decía: "Vamos a estudiar Italia. Tenemos que traer imágenes de Italia”.

\section{Folio giratorio}

En esta actividad, se pidieron imágenes sobre Italia (en la realización de la nota), las cuales tenían que poner en los murales. Se realizó juntando dos parejas, formando así, equipos de cuatro (dos alumnos de primaria y dos alumnos de infantil). Cada pareja tenía que decidir qué imagen quería poner en el mural; el alumno de infantil ponía el pegamento y la pegaba, y el alumno de primaria escribía el nombre de la imagen en la cartulina (por ejemplo, Torre de Pisa). Para finalizar, cada equipo explicó al resto de compañeros lo que habían puesto en su mural.

2 Conviene apuntar que la tutorización (de un alumno de primaria a un alumno de infantil) se ha realizado de manera combinada con las técnicas de: el folio giratorio, la adaptación de la técnica 1-2-4 y el Juego-Concurso de De Vries. 


\section{Adaptación técnica 1-2-4}

Se adaptó la técnica 1-2-4 pasando a ser la técnica 2-4-6, ya que el alumnado de infantil no podía realizar la parte individual. Además, se trabaja por parejas en todo momento (uno de infantil con uno de primaria).

La actividad consistía en pensar cosas referidas a lo trabajado sobre Europa (países, monumentos, comidas típicas, animales...) y escribirlas en la casilla correspondiente (la parte de escritura, únicamente fue realizada por el alumnado de primaria). En este caso, primero se trabajó por parejas (2), uno de infantil con uno de primaria, después se juntaron dos parejas (4), siendo: dos de infantil y dos de primaria, y por último, se juntaron 3 parejas (6) siendo: tres de infantil y tres de primaria.

\section{Juego-Concurso de De Vries}

Se realizó a través del famoso juego de "Pasapalabra", fabricado por el profesorado previamente. Para este juego, se agruparon por parejas (uno de infantil con uno de primaria) y se dividió la clase en dos grupos, realizando la actividad en dos sesiones. Se les facilitó preguntas con dos niveles de dificultad para cada letra del abecedario, para dar oportunidad de contestar tanto al alumnado de infantil como al de primaria. La pareja de alumnos se ponía dentro del rosco para poder responder. Primero, se realizaba la pregunta al de infantil y después al de primaria, si la contestaban correctamente se les daba la letra correspondiente en color verde, sino de color rojo. El objetivo era que entre todo el grupo-clase consiguieran realizar todo el rosco completo de manera óptima.

\subsection{Seguimiento del proceso y evaluación de la acción: recogida de evidencias}

En este punto se explica todo el proceso seguido para la recogida de datos, con el fin de evaluar el proceso de investigación-acción. Se han intentado obtener los datos desde diferentes perspectivas e instrumentos, para asegurarse que la información recogida tenga suficiente credibilidad (Guba, 1989) garantizando una triangulación de los datos (Aguilar y Barroso, 2015). Así pues, se llevó a cabo una observación participante durante el transcurso de todo el proyecto, una entrevista final con la tutora del aula y un análisis del material o documental. Asimismo, con el propósito de recoger la voz del alumnado protagonista del proyecto se realizó la dinámica "felicito- critico- propongo" y poder así evaluar todo el proceso realizado.

\subsubsection{Observación participante}

En esta fase del proceso se perpetró una observación participante. Según Fuertes (2011), dicha observación tiene lugar cuando el investigador o la persona que observa se involucra y "vive" las experiencias en el contexto y en el ambiente cotidiano de los sujetos, de modo que recoge los datos en tiempo real. Al igual que en la observación inicial se tomaron como referencia diferentes ítems de la Guía CEIN (Sales, Moliner y Traver, 2010) y se valoró su consecución en una tabla de registro. Además, se han ido recogiendo notas de campo, es decir, apuntes realizados de manera inmediata sobre la acción que se deseaba observar. 


\subsubsection{Entrevista final a la docente}

Por otra parte, se ejecutó una entrevista semiestructurada (con cierta flexibilidad) a la docente del aula de infantil, sobre el desarrollo del proyecto, para obtener su punto de vista sobre la propuesta articulada, siendo esta una gran fuente de información.

\subsubsection{Análisis documental}

El análisis documental fue utilizado para examinar los diferentes documentos o materiales originados desde la propia práctica educativa (Por ejemplo, notas escritas por el alumnado, mural de Italia, fichas actividad 2-4-6, escritos de la técnica 'Felicito, Critico, Propongo', entre otros). El análisis documental o material consiste en indagar sobre lo que Martínez Bonafé (1988) designa como evidencias documentales.

\subsection{4. 'Felicito, critico, propongo'}

Se utilizó una dinámica propia del diagnóstico social participativo mediante la técnica 'Felicito, critico, propongo' (Aguirre et al., 2018). A través de esta herramienta, se trató de evaluar únicamente las actividades que se habían realizado mediante las técnicas cooperativas, permitiendo valorar, en cierta manera, la satisfacción de los participantes, es decir, de los alumnos tanto de infantil como los de primaria.

Para ello, se juntó la mitad de la clase de primaria y la mitad de la clase de infantil, y se agruparon con sus respectivas parejas. El alumnado disponía de tres post-it's, uno de cada color (azul, naranja y amarillo). De esta manera, por parejas tenían que pensar, poniéndose de acuerdo entre ellos, en qué es lo que más les había gustado del proyecto y escribirlo en el post-it azul. A continuación, tenían que pegarlo en un papel continuo dividido en tres columnas, en su lugar correspondiente ('Felicito'). Del mismo modo lo hicieron con el post-it naranja, pero esta vez poniendo lo que menos les había gustado ('Critico'). Y, por último, siguiendo la misma dinámica, escribieron en un post-it amarillo las propuestas de mejora ('Propongo').

\section{Resultados}

Los resultados del estudio se presentan siguiendo el principal objetivo que ha guiado este trabajo centrado en analizar la incorporación de estructuras cooperativas a un proyecto internivel desarrollado con alumnado de infantil y primaria.

Primeramente, conviene apuntar que la planificación y puesta en acción de las actividades del proyecto llevado a cabo se han realizado mediante un proceso de investigación-acción, lo que permite partir de las necesidades del contexto. A continuación en la figura 1, se presenta un resumen del proceso 
efectuado.

\begin{tabular}{|c|}
\hline Fase 1: Detección de necesidades (diagnóstico situacional) \\
\hline 1.1. Entrevista inicial a la docente \\
1.2. Observación no participante \\
1.3. Anecdotario o notas de campo \\
\hline Fase 2: Planificación y puesta en acción \\
2.1. Tutorización \\
2.2. Folio giratorio \\
2.3. Adaptación técnica 1-2-4 \\
2.4. Juego-Concurso de De Vries \\
\hline Fase 3: Seguimiento del proceso y evaluación: recogida de evidencias \\
3.1. Observación participante \\
3.2. Entrevista final a la docente \\
3.3. Análisis documental \\
3.4. 'Felicito, critico, propongo' \\
\hline Fase 4: Propuestas de mejora (nueva propuesta de acción) \\
\hline
\end{tabular}

Figura 1. Resumen del proceso de investigación-acción realizado.

Con el fin de mostrar los resultados que se desprenden de este estudio, se van a presentar las diferentes evidencias recogidas mediante los instrumentos definidos anteriormente. Los resultados se presentarán siguiendo una serie de categorías o bloques de contenido. Asimismo, se identifica con un código la procedencia del dato, siendo: Observación (O), Notas de campo (NC), Entrevista (E) y Técnica 'Felicito, critico, propongo' (FCP).

Los agrupamientos por parejas, combinando alumnos de ambas clases, ha resultado ser un estupendo elemento motivador para el proyecto. "Todo el alumnado en general, estaba muy motivado con las actividades cooperativas, les gustó mucho trabajar juntos" (E). "Durante todas las sesiones, las dos aulas mostraban mucha alegría y motivación cuando se juntaban" (O). Hay que resaltar especialmente la figura que jugaban los alumnos del aula de educación primaria en la tutorización, ellos se sentían responsables y cuidadosos con los alumnos de infantil que tutorizaban. "El alumnado de primaria tenía una actitud muy positiva, se sentían felices y realizados ayudando a los más pequeños" (O). 
Generalmente, en lo que respecta a las actividades propiamente dichas llevadas a cabo en el proyecto, la actividad que más gustó al alumnado fue: "la de hacer y comer crepes" (FCP), correspondiente al bloque de contenido sobre la gastronomía de Francia. Por contra, la actividad que menos gustó al alumnado de primaria fue: "la de tomar el té" (FCP), cuando se trabajó la gastronomía de Londres. No obstante, para el alumnado de infantil fue una actividad en la que mostraron gran entusiasmo.

Refiriéndonos a la metodología y las actividades, decir que algunas técnicas como la adaptación de la técnica 2-4-6 han resultado complicadas, sobre todo para el alumnado de infantil. "En varias ocasiones se ha detectado al alumnado de infantil despistado, sin saber muy bien qué tenían que hacer" (NC). Además, en cuanto a la gestión del tiempo y el espacio también ha habido algunas deficiencias. "En algunos momentos el tiempo y el espacio no han sido adecuados, a causa de los espacios reducidos e inflexibilidad del horario de primaria" (E). Por lo general, la tutorización ha sido eficaz, aunque: "en la mayoría de actividades realizadas bajo técnicas cooperativas, eran los alumnos de primaria los que se encargaron de hacerlas" (E). Así pues, en referencia a los objetivos y contenidos del proyecto decir que en la mayoría de las sesiones, algunos objetivos y contenidos no eran asequibles para todo el alumnado. "Las técnicas eran un poco complicadas para los de infantil" (E). Se pudo observar, lógicamente, que: "el alumnado de infantil actuaba, en gran medida, influenciado por el de primaria" (NC). En la mayoría de las actividades, una de las principales críticas corresponde a que: "las opiniones procedían del alumnado de primaria, el de infantil no participaba en la respuesta" (FCP), debido a su nivel madurativo. "El alumnado de infantil, no estaba preparado para realizar ciertas actividades" (O).

\section{Nueva propuesta de acción}

Para finalizar el estudio, se plantean algunas propuestas de mejora que se podrían llevar a cabo para conseguir una mejor aplicación.

Por una parte, lo primero a mejorar sería el tiempo y el espacio. Se ha dispuesto, en la mayoría de casos, de espacios pequeños y un número elevado de alumnos. Por este motivo, en un futuro, se podrían formar grupos más pequeños para poder atender a todos de la mejor manera posible y potenciar un clima de aula más idóneo para trabajar. También, se plantearían las sesiones con una mayor duración, sin estar pendiente del horario, que por la parte de infantil no había problema, ya que tienen más flexibilidad, pero en cambio primaria tiene un horario más rígido, por lo que ha sido difícil cuadrar las sesiones.

A causa de falta de tiempo, se ha carecido de la participación de las familias, se podría haber solicitado la ayuda y la participación de estas.

Por otra parte, la edad de los participantes ha demostrado ser un factor determinante para la ade- 
cuada realización de las técnicas, pero no ha sido un inconveniente, ya que se han intentado ajustar las actividades acorde a su edad. En futuras aplicaciones se recomendaría realizar una pequeña formación a los alumnos de primaria centrada en “¿cómo ser buenos tutores?” para evitar que ellos hagan toda la actividad y ver cómo pueden mejorar sus habilidades como tutores. También se considera adecuado indagar sobre qué otras técnicas de aprendizaje cooperativo simples se podrían introducir para facilitar su uso en la tutorización del alumnado de primaria al de infantil.

\section{Discusión de resultados y conclusiones}

A modo de conclusión general, se puede sacar en claro que la mayoría de actividades del proyecto han sido valoradas muy positivamente y han dado el resultado esperado. En cambio, las actividades basadas en las técnicas cooperativas, han sido difíciles de llevar a cabo de manera óptima, debido a los diferentes ritmos y niveles madurativos del alumnado. En este trabajo se ha podido comprobar que la gestión pedagógica de la diversidad (Bergeron, 2015; Pujolàs, 2008; Tomlinson, 2005; Sanahuja, 2017) no es una tarea fácil y requiere de docentes comprometidos y reflexivos (Rosales, 1990) que vayan caminando hacia la mejora de sus propias prácticas docentes.

En cuanto a la técnica de la tutorización (la redacción de la nota entre el alumnado de infantil y primaria), a pesar del gran número de alumnado de ambas aulas (45 en total), disponer de un espacio reducido para tantos alumnos y tener un tiempo limitado en cada una de las sesiones, se creó un buen ambiente de trabajo. En todas las sesiones compartidas entre ambas clases, el alumnado de infantil tenía como modelo al alumnado de primaria en cuanto a actitud, comportamiento y realización de la actividad, pudiéndose efectuar satisfactoriamente. La cual cosa corrobora que la tutorización entre iguales resulta muy beneficiosa para los implicados (Durán, 2004; Moliner et al., 2015). Sin embargo, en la técnica del folio giratorio uno de los principales problemas que se detectaron en esta dinámica fue la incapacidad de espera, es decir, la mayoría de parejas de los grupos no esperaban a que la otra pareja colocara la imagen, y con ello a que girara el "folio" (la cartulina), sino que lo hacían a la vez. Con lo cual, la dinámica de dicha técnica no se asimiló. Es por ello que se considera de tan importancia trabajar previamente, a la introducción de las técnicas de aprendizaje cooperativo, las habilidades sociales referentes al ámbito A- cohesión de grupo propuestas en Pujolàs y Lago (2002). Con respecto a la adaptación de la técnica 1-2-4, no se logró la participación de todos por igual. En algunos casos, los alumnos de primaria realizaban la actividad, quedando en un segundo plano el alumnado de infantil, es decir, adoptando un papel más pasivo. Este indicio nos vuelve a remitir a la importancia de que el tutor aprenda a ser un buen tutor. Sin embargo, en otros casos su funcionamiento fue adecuado, dado que el alumnado de primaria ya había utilizado esta técnica. Por último, en cuanto al Juego-Concurso de De Vries, llevado a cabo mediante el juego de 'Pasapalabra', dio resultados muy positivos. El alumnado 
estaba motivado, ya que conocían el juego y les resultó muy divertido. La dinámica fue muy adecuada, ya que se hizo la actividad por pequeños grupos, para evitar tiempos largos de espera, aburrimiento y/o desmotivación. Además, permitió evaluar de forma efectiva al alumnado y se pudo ver todos los conocimientos que habían adquirido.

En cuanto a la incorporación de estructuras cooperativas a un proyecto internivel desarrollado con alumnado de infantil y primaria, podemos concluir que las técnicas cooperativas aplicadas a niños de infantil de tres años, tienen un alto grado de complejidad. Se puede decir que la edad de los participantes ha sido un factor muy determinante para la correcta consecución de las actividades. Al ser niños tan pequeños, aunque estén tutorizados por alumnos de segundo de primaria, no están lo suficientemente maduros para realizar dichas técnicas. Esto hace que el alumnado de infantil no haya sido tan partícipe como se desearía en la ejecución de estas. Esto mismo ha ocurrido en la dinámica 'Felicito, critico, propongo' (Aguirre et al., 2018), la cual resultó un poco complicada para recoger las voces del alumnado, sobre todo del de infantil.

A modo de conclusión general podemos apuntar que los objetivos marcados en este estudio se han cumplido y gracias al proceso de investigación- acción se ha podido reflexionar sobre la práctica educativa llevada a cabo con el fin de mejorarla. No obstante, se considera adecuado continuar trabajando e introduciendo poco a poco diferentes técnicas simples de aprendizaje cooperativo en el segundo ciclo de educación infantil. A menudo, se realizan experiencias aisladas o esporádicas que van en detrimento de los principios y valores que persigue una educación basada en la inclusión y en la participación democrática. Por ello, se necesita a docentes comprometidos que apuesten por esta forma de trabajar en el aula.

\section{Referencias}

Aguilar, S. y Barroso, J. (2015). La triangulación de datos como estrategia en investigación educativa. Revista de Medios y Educación, 47, 73-88. Disponible en: https://recyt.fecyt.es/index.php/pixel/article/view/61672

Aguirre, A., Benet, A., Moliner, O., Sales, A., Sanahuja, A., Traver, J.A. (2018). Técnicas para la participación democrática. La formación para la convivencia democrática en los procesos de mejora. Castellón de la Plana (España): Servicio de Publicación de la Universitat Jaume I.

Apple, M. W. y Beane, J. A. (1999). Escuelas democráticas. Madrid (España): Morata.

Araujo, U.F. y Sastre, G. (2008). El Aprendizaje Basado en Problemas. Una nueva perspectiva de la enseñanza en la Universidad. Barcelona: Gedisa. 
Aylwin, U. (1992). La pédagogie différenciée fait son entrée au collège. Pédagogie collégiale, 5(3), 30-37. Disponible en: https://cdc.qc.ca/ped coll/pdf/aylwin ulric 05 3.pdf

Báez, J. (2009). Investigación cualitativa. Madrid (España): ESIC EDITORIAL.

Balongo, E. y Mérida, R. (2016). El clima de aula en los proyectos de trabajo. Crear ambientes de aprendizaje para incluir la diversidad infantil. Perfiles Educativos, 37 (152), 146162. Disponible en: http://www.scielo.org.mx/pdf/peredu/v38n152/0185-2698-peredu-38-152-00146.pdf

Barrio, J.L. (2008). Hacia una Educación Inclusiva para todos. Revista Complutense de Educación, 20 (1), 13-31. Disponible en: http://revistas.ucm.es/index.php/RCED/article/view/RCED0909120013A/15360

Batlle, R. (2013). El aprendizaje servido en España: el contagio de una revolución pedagógica. Madrid (España): PPC.

Bender, W.N. (2012). Differentiating Instruction for Students With Learning Disabilities. California: Corwin A SAGE Company.

Benet, A., Sanahuja, A. y Moliner, L. (2016). Rincones, from the teachers' perspectives: a reflection on a primary education practice. 9th International Conference of Education, Research and Innovation, Sevilla (Espanya).

Bergeron, L. (2015). La planification de l'enseignement a priori en fonction de la diversité des élèves. Une logique préventive et proactive. In N. Rosseau (dir.), La pédagogie de l'inclusion scolaire: Un défi ambitieux et stimulant (pp.375-397). Québec (Canada): Presses de l’Université du Québec.

Campbell, B. (2008). Handbook of Differentiated Instruction using the Multiple Intelligences. Lessons Plans \& More. Boston: Pearson Allyn \& Bacon.

Caron, J. (2003). Apprivoiser les différences : guide sur la différenciation des apprentissages et la gestion des cycles. Montréal (Canada) : Éditions de la Chenelière.

Cerdá, M.C. e Iyanga, A. (2013). Evolución legislativa de la educación especial en España de 1970 a 2006 y su aplicación práctica. Revista nacional e internacional de educación inclusiva, 6 (3), 150-163. Disponible en:

http://www.revistaeducacioninclusiva.es/index.php/REI/article/view/157 
Durán, D. (2004). Tutoría entre iguales. Innovación Educativa, 75 , 63-68. Disponible en: http:// grupsderecerca.uab.cat/grai/sites/grupsderecerca.uab.cat.grai/files/monograficoaula.pdf

Echeita, G. (2013). Inclusión y exclusión educativa. De nuevo "voz y quebranto”. Revista Iberoamericana sobre Calidad, Eficacia y Cambio en Educación, 11 (2), 99-118. Disponible en: http://www.redalyc.org/pdf/551/55127024005.pdf

Echeita, G. y Ainscow, M. (2011). La Educación inclusiva como derecho. Marco de referencia y pautas de acción para el desarrollo de una revolución pendiente. Tejuelo, 12, 26-46. Disponible en: https://repositorio.uam.es/handle/10486/661330

Elboj, C. \& Niemelä, R. (2010). Sub-Communities of Mutual Learners in the Classroom: The case of Interactive Groups. Revista de Psicodidáctica. 15 (2) 177-189. Disponible en: http:// www.redalyc.org/pdf/175/17517246003.pdf

Feito, R. (2010). Escuela y democracia. Política y sociedad, 47 (2), 47-61.

Feito, R. y López, J.I. (2008).Construyendo escuelas democráticas. Barcelona (España): Hipatia.

Fillion, P.L., Bergeron, G., Prud'homme, L. y Traver, J. (2016). L'éducation à la citoyenneté démocratique: un enjeu fondamental associé au projet d'inclusion scolaire et aux pratiques de différenciation pédagogique. In L. Prud'homme, H. Duchesne, P. Bonvin, \& R. Vienneau (Eds.), L'inclusion scolaire : ses fondements, ses acteurs et ses pratiques. Bruxelles : De Boeck Supérieur.

Forsten, C., Grant, J. \& Hollas, B. (2002). Differentiated instruction : different strategies for different learners. Peterborough : Crystal Spring Books.

Fuertes, $M^{\mathrm{a}}$ T. (2011). La observación de las prácticas educativas como elemento de evaluación y de mejora de la calidad en la formación inicial y continua del profesorado. Revista de Docencia Universitaria, 9 (3), 237-258. Disponible en: http://red-u.net/redu/files/journals/1/articles/248/public/248-647-1-PB.pdf

Gardner, H. (1983). Multiple Intelligences: The Theory and Practice. New York: Basic Books.

Gregory, G.H. \& Chapman, C. (2013). Differentiated Instructional Strategies: One Size Doesn't Fit All. Thousand Oaks, California: Corwin A SAGE Company. 
Guay, M.H., Legault, G. et Germain, C. (2006). Pour tenir compte de chacun : La différencia tion pédagogique, Vie Pédagogique. 141, 1-4. Disponible en: http://vmedulnx01.uqo.ca/moreau/documents/Guay2006.pdf

Guba, E. G. (1989). Criterios de credibilidad en la investigación naturalista. En J. Gimeno y A. Pérez (Comp.). La enseñanza: su teoría y su práctica (pp.148-165). Madrid: Akal.

Heacox, D. (2002). Differentiating Instruction in the Regular Classroom: How to Reach and Teach All Learners, Grades 3-12. Minneapolis: Free Spirit.

Jiménez, F. y Vilá, M. (1999). De educación especial a la educación a la diversidad. Málaga: Aljibe.

Kline, B. (2015). Content, Process, and Product: Modeling Differentiated Instruction. Kappa Delta Pi Record 51 (1), 13-17. Disponible en: https://www.tandfonline.com/doi/abs/10.1080/00228958.2015.988559

Latorre, A. (2003). La investigación-acción. Conocer y cambiar la práctica educativa. Barcelona, (España): Graó.

Legendre, R. (2005). Dictionnaire actuel de l'éducation. Montréal (Canada): Guérin.

Legrand, L. (1995). Les différenciations de la pédagogie. Paris (Francia): PUF.

Levy, H.M. (2008). Meeting the needs of all students through differentiated instruction: helping every child reach and exceed standards. Clearing House: A Journal of Educational Strategies, Issues and Ideas, 81(4), 161-164 Disponible en: http://www.wou.edu/ tbolsta/web/texbook/24 Meeting the Needs.pdf

Lira, R.I. (2010). Las metodologías activas y el foro presencial: su contribución al desarrollo del pensamiento crítico. Revista Actualidades Investigativas en Educación, 10 (1), 1-18 Disponible en: http://www.redalyc.org/articulo.oa?id=44713068008

López, A.M. y Lacueva, A. (2007). Proyectos en el aula: cinco categorías en el análisis de un caso. REICE: Revista Electrónica Iberoamericana sobre Calidad, Eficacia y Cambio en Educación, 5 (1), 78-120. Disponible en: http://www.redalyc.org/pdf/551/55100106.pdf

Martínez Bonafé, J. (1988). El estudio de caso en la investigación educativa. Revista Investigación en la Escuela, 6, 41-50. Disponible en: https://idus.us.es/xmlui/handle/11441/59162 
Mayordomo, R. Ma. y Onrubia, J. (2015). El aprendizaje cooperativo. Elementos conceptuales. En R. Ma Mayordomo y J. Onrubia (Coords.). El aprendizaje cooperativo. (pp. 17-48). Barcelona (España): Editorial UOC.

Meirieu, P. (1996). Itinéraire des pédagogies de groupe : apprendre en groupe. Lyon : Chronique sociale.

Ministerio de Educación, Cultura Deporte (2011). Educación inclusiva en el sistema educativo - Mecd.gob.es. Disponible en: https://www.mecd.gob.es/educacion-mecd/areaseducacion/estudiantes/educacion-inclusiva/necesidad-apoyo-educativo.html:jsessionid $=2 \mathrm{E} 212290 \mathrm{C} 014 \mathrm{~A} 33505 \mathrm{~EB} 0 \mathrm{E} 1066487407$

Moliner, A. y Sanahuja, A. (2015). El pas del temps a Betxí: una experiència de treball per projectes en educació primària. Fòrum de recerca, núm. 20, 479-499. Disponible en: http:// repositori.uji.es/xmlui/handle/10234/172621

Moliner, O., Sanahuja, A. y Benet, A. (2017). Prácticas inclusivas en el aula desde la investigaciónacción. Colección Sapientia 127. Castellón de la Plana, España: Servicio de Publicación de la Universitat Jaume I.

Moliner. O., Moliner, L., Sanahuja, A. y Sanmateo, V. (2015). Análisis de los elementos de la tutoría entre iguales que posibilitan avanzar hacia la construcción de una escuela intercultural inclusiva y democrática. Revista Latinoamericana de Inclusión Educativa, 9(2), 41-58. Disponible en: http://repositori.uji.es/xmlui/handle/10234/153831

Muntaner J. J. (2013). Calidad de vida en la escuela inclusiva. Revista iberoamericana de educación, (63), 35-49. Disponible en: https://rieoei.org/RIE/article/view/421

Perrenoud, P. (1996). La pédagogie à l'école des différences. Paris (Francia): EFS.

Prud'homme, L., Paré, M., Leblanc, M., Bergeron, G., Sermier-Dessemontet, R., \& Noël, I. (2016). La différenciation pédagogique dans une perspective inclusive : quand les connaissances issues de la recherche rencontrent le projet d'éducation pour tous. In L. Prud'homme, H. Duchesne, P. Bonvin, \& R. Vienneau (Eds.), L'inclusion scolaire : ses fondements, ses acteurs et ses pratiques. Bruxelles : De Boeck Supérieur.

Pujolàs, P. (2008). El aprendizaje cooperativo. Barcelona, España: GRAÓ. 
Pujolàs, P. y Lago, J. R. (Coord.) (2002). El programa CA/AC (“cooperar para aprender/aprender a cooperar”) para enseñar a aprender en equipo Implementación del aprendizaje cooperativo en el aula. Universidad de Vic.

Rosales, R. (1990). Evaluar es reflexionar sobre la enseñanza. Madrid (España): Narcea Ediciones.

Rué, J. (1998). L'immigrant a l'escola. La metodologia del treball cooperatiu com a via d'integració social i acadèmica dels alumnes d'origen immigrant. Educar, 22-23. Disponible en: http://www.raco.cat/index.php/Educar/article/download/20695/20535

Ruiz Olabuénaga, J.I. (2012). Metodología de la investigación cualitativa. Bilbao (España): Deusto.

Ruiz, Ma . C. y Mérida, R. (2014). El desarrollo profesional docente derivado de la aplicación de los Proyectos de Trabajo en un aula infantil. Un estudio de caso. Revista Complutense de Educación, 27 (2), 477-497. Disponible en: https://revistas.ucm.es/index.php/RCED/ article/viewFile/46378/48387

Saint-Laurent, L. (2007). Enseigner aux élèves à risque et en difficulté au primaire. Montréal : Éditions de la Chenelière.

Sales, A., Moliner, O. y Traver, J. (2010). La construcción de la escuela intercultural inclusiva desde procesos de investigación-acción. Castelló (España): Servicio de Publicaciones de la Universitat Jaume I.

Sanahuja, A. (2014). Grupos interactivos: propuesta de estructuración para el aula de infantil y primaria. I Congreso Internacional de Necesidades Específicas de Apoyo Educativo, Universidad de Almería.

Sanahuja, A. (2017). Diferenciación pedagógica y participación democrática en el aula inclusiva: estudio de casos múltiples (Tesis Doctoral). Universitat Jaume I, Castellón de la Plana.

Sanahuja, A. Peiro, M. y Piquer, A. (2015). La prehistoria: una experiencia de trabajo por proyectos en educación primaria. Quaderns Digitals, no 81. 6-23. Disponible en: http://www. quadernsdigitals.net/index.php?accionMenu=hemeroteca.VisualizaArticuloIU. visualiza\&articulo id=11419

Sanahuja, A. y Moliner, O. (2018). Aprenentatge-Servei al màster en psicopedagogia de l'UJI: una experiència d'innovació educativa. IV Jornada d'experiències d'innovació educativa (JEIE2018). Universitat Jaume I, Castellón (España). 
Sanahuja, A., Benet, A. y Traver, J.A. (2017). La tertulia pedagógica dialógica: un cambio de mirada en el perfil del psicopedagogo/a. XIV Congreso Internacional de Teoría de la Educación. Murcia (España).

Sandín. M.P. (2003). Investigación Cualitativa en Educación. Fundamentos y Tradiciones. Madrid, España: McGraw Hill.

Santos, s.f. El Aprendizaje cooperativo en la enseñanza universitaria. Recuperado de http://www.ice.uib.cat/digitalAssets/180/180395 act 32.pdf

Stake, R.E. (1998). Investigación con estudio de casos. Madrid, España: Morata.

Subban, P. (2006). Differentiated instruction: A research basis. International Education Journal, 7(7), 935-947. Disponible en: https://files.eric.ed.gov/fulltext/EJ854351.pdf

Tomlinson, C.A. (2005). Estrategias para trabajar con la diversidad en el aula. Buenos Aires, Argentina: Paidós.

Traver, J.A. (2000). Trabajo cooperativo y aprendizaje solidario: Aplicación de la técnica puzzle de Aronson para la enseñanza y el aprendizaje de la actitud de solidaridad. (Tesis doctoral). Universitat Jaume I, Castellón, España.

Vázquez, J. (2004). La observación científica en el proceso de contrastación de hipótesis y teorías. Theoria: Revista de Teoría, Historia y Fundamentos de la Ciencia 19 (1), 77-95. Disponible en: https://philpapers.org/rec/VZQLOC-2 